\title{
Implementing Collaborative Care Management of Behavioral Health for Patients with Inflammatory Bowel Disease
}

\author{
Christine B. Flicek $^{a}$ Nathaniel A. Sowa ${ }^{a}$ Millie D. Long ${ }^{b}$ Hans H. Herfarth ${ }^{b}$ \\ Spencer D. Dorn ${ }^{b}$

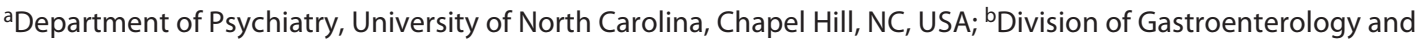 \\ Hepatology, Department of Medicine, University of North Carolina, Chapel Hill, NC, USA
}

\section{Keywords}

Inflammatory bowel disease $\cdot$ Collaborative care .

Depression · Anxiety · Behavioral health

\begin{abstract}
Background: Individuals with inflammatory bowel disease (IBD) are up to twice as likely to suffer from anxiety and/or depression. Collaborative care management (CoCM) is an evidence-based approach to treating behavioral health disorders that have proven effective for a range of conditions in primary care and some specialty settings. This model involves a team-based approach, with care delivered by a care manager (case reviews and behavioral therapy), psychiatrist (case reviews and psychopharmacological recommendations), and medical provider (ongoing care including psychopharmacological prescriptions). We assessed the feasibility and effectiveness of CoCM in reducing anxiety and depressive symptoms in patients with IBD. Methods: Patients with psychological distress identified by clinical impression and/or the results of the Patient Health Questionaire-9 (PHQ9) and Generalized Anxiety Disorder-7 (GAD-7) were referred to the CoCM program. Data from our 9-month CoCM pilot were collected to assess depression and anxiety response
\end{abstract}

karger@karger.com www.karger.com/iid

Karger $\stackrel{\text { ' }}{5}$

BOPEN ACCESS
(C) 2021 The Author(s)

Published by S. Karger AG, Basel

This is an Open Access article licensed under the Creative Commons Attribution-NonCommercial-4.0 International License (CC BY-NC) (http://www.karger.com/Services/OpenAccessLicense), applicable to the online version of the article only. Usage and distribution for commercial purposes requires written permission. and remission rates. We obtained provider surveys to assess provider acceptability with delivering care in this model. $\boldsymbol{R e}$ sults: Though the SARS-CoV2 COVID-19 pandemic interrupted screening, 39 patients enrolled and 19 active participants completed the program. Overall, $47.4 \%$ had either a response or remission in depression, while $36.8 \%$ had response or remission in anxiety. The gastroenterologists highly agreed that the program was a beneficial resource for their patients and felt comfortable implementing the recommendations. Discussion: CoCM is a potentially feasible and well accepted care delivery model for treatment of depression and anxiety in patients with IBD in a specialty gastroenterology clinic setting.

(C) 2021 The Author(s).

Published by S. Karger AG, Basel

\section{Introduction}

Patients with inflammatory bowel disease (IBD) have a higher risk of comorbid depression and anxiety compared to the general population [1-4]. Depression has been associated with more aggressive IBD and linked to increased recurrence of disease [5-7]. Compared to patients with IBD and no psychiatric illness, those with co- 
morbid behavioral health concerns suffer lower quality of life and increased pain, are less adherent and responsive to therapy, are at increased risk of requiring surgery, and have higher health care utilization [4, 8-10]. Furthermore, in a large population-based study, use of antidepressants has been found to have a beneficial impact on the disease course among patients with IBD, particularly in patients with no use of antidepressants before IBD onset [11]. Due to these associations, there is an increased emphasis on the importance of behavioral health screening as part of IBD treatment $[12,13]$. However, many gastroenterologists lack the training and resources needed to identify and manage behavioral health concerns [12].

The collaborative care management (CoCM) model may be a more effective approach for integrating behavioral health into IBD care. CoCM is a patient-centered, team-based approach involving collaboration between the medical provider and behavioral health providers, including a psychiatric care manager and a psychiatric consultant [14]. The model delivers population- and measurement-based treatment to target by tracking all enrolled patients in a registry, using evidence-based patient-reported outcome measures to assess symptoms and monitor clinical outcomes to guide treatment plan changes. The patient works directly with the psychiatric care manager on behavioral interventions. Psychotropic medications, if clinically indicated, are managed by the medical provider with the guidance and recommendations of a psychiatric consultant. Through more than 90 randomized controlled studies in both primary care and specialty settings, CoCM has proven to improve patient outcomes, reduce anxiety and depression, improve quality of life, increase both patient and provider satisfaction, and reduce health care costs [14-16].

Here, we describe the implementation of $\mathrm{CoCM}$ for patients with IBD. Though there have been other integrated care approaches to address the need for behavioral health access, such as direct patient care by a behavioral health provider imbedded in an IBD clinic, to our knowledge, this is the first description of implementation of CoCM for this population [17].

\section{Methods}

\section{Study Setting and Enrollment Criteria}

The CoCM (described below) was implemented within a large academic center adult IBD clinic with 8 IBD providers. The clinic used 2 strategies to recruit patients for enrollment between December 2019 and August 2020. First, the 8 IBD-focused gastroenterologists used their clinical judgment to identify and refer both

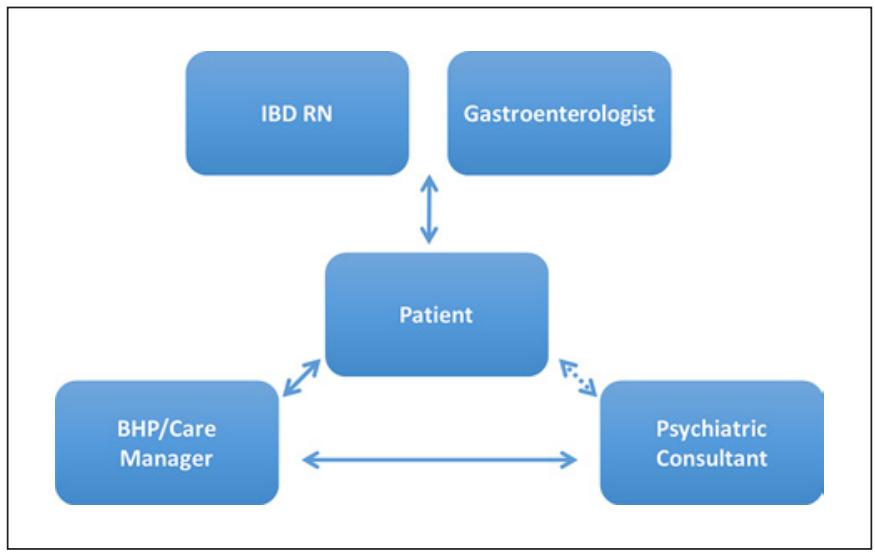

Fig. 1. Patient-centered and team-based approach in collaborative care.

new and established patients with reported behavioral health concerns. Second, at each clinic visit between December 2019 and March 12, 2020 (when in-person visits were largely stopped due to the SARS-CoV2 pandemic), the Patient Health Questionaire-9 (PHQ-9) and Generalized Anxiety Disorder-7 item (GAD-7) scales were used to screen all new and established patients for depression and anxiety symptoms, respectively. Both scales assess recent symptoms within the past 2 weeks and use similar cutoff ranges to reflect level of symptoms ranging from mild (5-9), moderate $(10-14)$, and scores greater than 15 indicating severe symptoms $[18,19]$. Those with at least moderate level of depressive or anxiety symptoms, as defined by PHQ-9 or GAD-7 greater than or equal to 10 , were offered referral to the program $[18,19]$. All patients were 18 years or older and spoke fluent English.

Patients were enrolled in the program if they completed an initial behavioral assessment with the care manager and completed the treatment care plan. Patients were disenrolled if they were identified as requiring a higher level of care than CoCM could provide or were not sufficiently engaged (i.e., 3 or more unsuccessful outreach attempts to the patient by the care manager). Patients who were disenrolled due to lack of engagement returned to the usual level of care in the GI clinic with referral resources. This report includes patients who had 2 or more case review sessions with the psychiatric consultant and were not disenrolled due to lack of engagement (heretofore referred to as "active participants"). Importantly, the COVID-19 pandemic severely restricted the number of patients seen in-person in the clinic, thereby eliminating PHQ9 and GAD-7 screening for psychological distress (which were only done in-person). As a result, referrals were only made based on provider clinical judgment alone after March 12, 2020, which reduced the number of patients referred to the CoCM program.

\section{Intervention}

The intervention team included the primary gastroenterologist, the IBD specialty nurse, a psychiatric consultant, and a licensed clinical social worker as the care manager (Fig. 1). The intervention modeled the Improving Mood Promoting Access to Collaborative Treatment (IMPACT) collaborative care design developed by the University of Washington, which has been described in detail elsewhere [14].
Flicek/Sowa/Long/Herfarth/Dorn 
Table 1. Patient characteristics

\begin{tabular}{|c|c|c|c|}
\hline Male, $n(\%)$ & $8(20.5)$ & $5(31.3)$ & $3(15.8)$ \\
\hline Average age, year & 42 & 43 & 40 \\
\hline Racial minority, $n(\%)$ & $9(23.1)$ & $5(31.3)$ & $4(21.1)$ \\
\hline Crohn's disease & $30(76.9)$ & $14(87.5)$ & $13(68.4)$ \\
\hline Ulcerative colitis & $5(12.8)$ & $2(12.5)$ & $3(15.8)$ \\
\hline Pouchitis & $2(5.1)$ & $0(0.0)$ & $2(10.5)$ \\
\hline IBDU & $2(5.1)$ & $0(0.0)$ & $1(5.3)$ \\
\hline \multicolumn{4}{|c|}{ Psychiatric treatment care plan, $n$ (\%) } \\
\hline Depression & $13(33.3)$ & $7(43.8)$ & $6(31.6)$ \\
\hline GAD-7 (average) & $10.6(S D=5.0$, range $2-19)$ & $13.3^{*}(\mathrm{SD}=4.5$, range $5-19)$ & $8.9^{*}(\mathrm{SD}=4.9$, range $2-18)$ \\
\hline \multicolumn{4}{|l|}{ End of program PROs } \\
\hline PHQ-9 (average) & & & $7.9(\mathrm{SD}=5.8$, range $0-17)$ \\
\hline GAD-7 (average) & & & $6.7(S D=5.1$, range $0-17)$ \\
\hline
\end{tabular}

PRO, patient-reported outcome; IBDU, Inflammatory Bowel Disease Unclassified; SD, standard deviation. ${ }^{*} p<0.05$.

The care manager contacted subjects referred to the program by telephone or video and explained the CoCM program and answered questions. Upon enrollment, the care manager performed an initial assessment that included a review of current behavioral health symptoms, psychosocial factors, and past and current behavioral health treatment. Based on this assessment, the patient and care manager developed a treatment care plan for anxiety, depression, or both in line with the patient's goals for treatment.

The care manager attempted to connect with enrolled subjects via telephone or video outreach at least once every other week. During outreach, the care manager established treatment goals with the patient. Based on these goals, the care manager provided brief behavioral interventions, such as behavioral activation, problem-solving therapy, sleep hygiene education, distress tolerance skills, and motivational interviewing. The care manager also assessed for psychotropic medication adherence, inquired on any side effects of their medication regimen, and obtained updated PHQ-9 and GAD-7 scores when indicated.

Each week the psychiatrist and care manager met to review each participating patient, with special focus on patients who were more symptomatic and needed treatment plan adjustments. The psychiatrist, based on chart review and information gathered from the care manager, documented medication, or behavioral recommendations in the electronic medical record for the gastroenterologist to consider. The care manager and/or gastroenterology nurse assisted with communication of the recommendations to the patient and served as liaisons between the patient and their gastroenterologist. The patient and their gastroenterologist ultimately determined which treatment recom- mendations to implement. The gastroenterologist prescribed all psychotropic medication prescriptions determined clinically indicated.

Patients who had a sustained response (based on $50 \%$ decrease in enrollment PHQ-9 and/or GAD-7 for 3 months) and/or who subjectively felt they had enough improvement graduated from the program. At time of graduation, the care manager completed relapse prevention plans with the patients.

The CoCM pilot provided treatment and collected patient outcome measures from December 2019 to September 2020. After this, patients who were still enrolled were provided transition plans, such as relapse prevention plans, final medication or behavioral recommendations, and/or referral to higher level of care if indicated.

\section{Measures}

Anxiety and Depression

PHQ-9 (depression) and GAD-7 (anxiety) scores were obtained during enrollment and subsequent scores were obtained every 3 months or earlier if clinically indicated. End of program scores, which included scores at time of graduation or most recent scores at time of program termination, were compared to baseline PHQ-9 and GAD-7 scores using a paired $t$ test to determine if there was a statistically significant difference. Patients were determined to have a response in treatment if their end of program scores were at least $50 \%$ lower than at baseline and remission if scores on either PHQ-9 or GAD-7 were less than 5 and not increased from baseline $[18,19]$. 
Provider Satisfaction

To determine gastroenterology provider satisfaction with the collaborative care program in the IBD clinic, each of the 8 attending gastroenterologists completed an anonymous online survey disseminated and analyzed using Qualtrics software. The survey included 10 statements addressing various aspects of the collaborative care program (with level of disagreement or agreement with each statement based on a 10-point Likert scale), as well as free-text feedback on the most useful aspects of the program and improvement suggestions.

\section{Results}

\section{Screening}

From January 2020 to March 2020, 364/699 (52.1\%) of all scheduled IBD clinic patients were screened for depression and anxiety symptoms. Of those screened, $65 / 364$ (17.9\%) scored $\geq 10$ on the PHQ-9 and/or GAD-7, indicating at least moderate levels of depressive and/or anxiety symptoms. Of those 65, 25 (38.5\%) agreed to referral to the program and 17 of the $25(68 \%)$ ultimately enrolled.

\section{Enrollment}

Overall, based on combination of referrals from screening or clinician judgment, 39 patients enrolled and completed an initial psychiatric treatment care plan with the care manager from December 2019 to August 2020. Enrollment dramatically declined after March 2020, when the ability to perform in-clinic behavioral health screening was interrupted due to the COVID-19 pandemic. 33/39 (85\%) were enrolled through March 2020, with only $6 / 39(15 \%)$ enrolled after the interruption in screening.

Nineteen patients remained in the program as active participants and were included in the outcome analysis. The 19 active participants had an average duration of program participation of 192.7 days (standard deviation 81.9, range: 42-306) and 6.7 psychiatric case reviews (standard deviation 2.9 , range: $3-11$ ).

Most enrolled and active participants were female, white, and had a diagnosis of Crohn's disease (Table 1). Compared to active participants, those who disenrolled due to lack of engagement had higher baseline PHQ-9 (10.1 vs. $13.1, p=0.15$ ) and GAD-7 (8.9 vs. $13.3, p=0.02$ ) scores.

\section{Antidepressant Use}

The majority of active participants were not on an antidepressant medication at baseline, with $42.1 \%(8 / 19)$ re-
Table 2. PRO measures of active patients $(n=19)$

\begin{tabular}{lll}
\hline Active participants & Depression & Anxiety \\
\hline Response, \% & $36.8(7 / 19)$ & $31.6(6 / 19)$ \\
Remission, \% & $31.6(6 / 19)$ & $26.3(5 / 19)$ \\
Response or remission, \% & $47.4(9 / 19)$ & $36.8(7 / 19)$ \\
\hline
\end{tabular}

Key: response $=>50 \%$ reduction in score from baseline, remission $=$ score $<5$ and not increased from baseline. PRO, patient-reported outcome.

porting use of at least 1 antidepressant medication at the time of enrollment. The psychiatric consultant recommended starting or adjusting the antidepressant dose for 78.9\% (15/19) participants, who had higher baseline PHQ-9 or GAD-7 scores compared to the participants where no change or initiation of an antidepressant was recommended (PHQ-9 $p=0.01$, GAD-7 $p=<0.01$ ). Of the 15 participants, the recommendation was implemented in $80 \%(12 / 15)$ based on patient report with $20 \%(3 / 15)$ stating preference for no medication changes. Twentyfive percent (3/12) discontinued their antidepressant by end of program due to combination of lack of tolerability or lack of perceived benefit. In total, at the end of the program participation, $57.9 \%(11 / 19)$ remained on an antidepressant regimen.

\section{Anxiety and Depression}

Although not statistically significant, program participants demonstrated numerical reductions in depression and anxiety scores between baseline and end of program scores (PHQ-9 $p=0.18$, GAD-7 $p=0.18$ ), with the average depression score changing from moderate $(\geq 10)$ to mild $(<10)$ level of depression severity. Depressive symptoms had a higher rate of percentage achieving response or remission compared to anxiety $(47.4 \%$ [9/19] compared to $36.8 \%$ (7/19). A slightly higher percentage of patients demonstrated a response and remission in depression symptoms compared to anxiety symptoms $(36.8 \%$ [7/19] vs. $31.6 \%[6 / 19]$ response; $31.6 \%$ [6/19] vs. $26.3 \%$ [5/19] remission, Table 2).

\section{Provider Satisfaction}

The 8 gastroenterology providers ranged from 1 to 25 years of clinical gastroenterology experience. All highly agreed that the collaborative care program was a beneficial resource for them and their patients, they were glad the program available and felt comfortable implementing the psychiatrist's recommendations (Table 3 ). 
Table 3. IBD provider satisfaction

\begin{tabular}{|c|c|c|}
\hline Survey statements & Mean & SD \\
\hline I feel comfortable implementing the recommendations made by the psychiatrist within the collaborative care program & 9.57 & 0.79 \\
\hline The collaborative care program has been a beneficial behavioral health resource to offer my patients & 9.5 & 1.41 \\
\hline I am able to provide improved clinical care for patients with mental health concerns with the resources of the collaborative care program & 9.13 & 1.81 \\
\hline I am satisfied with the services my patients receive in the behavioral health care management program & 9.13 & 1.81 \\
\hline The involvement of the collaborative care program has improved my satisfaction in caring for patients with mental health concerns & 8.88 & 1.81 \\
\hline My patients report to me that they are satisfied with the services they receive in the behavioral health care management program & 8.38 & 3.11 \\
\hline My knowledge base for treating patients with mental health disorders has increased within the collaborative care model & 8.25 & 2.71 \\
\hline $\begin{array}{l}\text { The involvement of the collaborative care program has improved my efficiency in providing clinical care to patients with mental health } \\
\text { concerns }\end{array}$ & 7.88 & 2.75 \\
\hline
\end{tabular}

Ten-point Likert scale with defied values of low (1-4), moderate (5-7), and high (8-10) agreement with statement. SD, standard deviation.

Their key future suggestions pertained to developing strategies to engage patients who do not respond to calls for outreach, as well as more clinic resources for counseling and increasing educational opportunities such as didactics on behavioral health strategies being utilized and group educational sessions (online suppl. Table 1; see www.karger.com/doi/10.1159/000521285 for all online suppl. material).

\section{Discussion}

We developed and implemented a CoCM program within an IBD specialty clinic with a goal of reducing comorbid anxiety and depression that is linked to poorer health outcomes and higher health care costs $[4,8-10]$. Nearly one-fifth $(65 / 364,18 \%)$ of the screened patients in our study population showed signs of moderate depression and anxiety and of those, $39 \%$ (25/65) agreed to enroll in a psychiatric treatment plan. Other patients were referred based on the gastroenterologist clinical impression. Of active participants, more than one-third (9/19, 47.4\% for depression and $7 / 19,36.8 \%$ for anxiety) showed response or remission in depression or anxiety. Recruitment and execution of this study were significantly impacted by the ongoing COVID-19 pandemic, but to our knowledge, this is the first description of implementation of CoCM in an IBD clinic to treat this specialty population.

In our pilot study, $18 \%$ of the screened patients showed moderate levels of anxiety and depression, which is in the same range as the prevalence of self-reported depression in a large USA-cohort study [6]. Lewis et al. [20] have reported that when more refined tools are utilized for detecting undiagnosed depression and anxiety in individuals with IBD, the prevalence is greater than $30 \%$. Consequently, the most recent American College of Gastroenterology treatment guidelines for ulcerative colitis incorporate the recognition and treatment of depression as a treatment goal [13]. Thus, to successfully build interventions, it is important to recognize concurrent depression and/or anxiety. A key component of CoCM includes using screening measures as part of routine workflow to identify these individuals. The PHQ-9 has good sensitivity and specificity for detecting depressive disorders and the GAD-7 has good operating characteristics for detecting generalized anxiety, panic, social anxiety, and posttraumatic stress disorder [18, 19, 21, 22].

Successes of our intervention in the IBD clinic included implementation of in-person screening for anxiety and depression in the clinic, which was previously not part of the workflow. In addition, we were able to successfully engage specialists in this novel approach to identify and treat patients with behavioral health concerns within their clinic. Overall, the gastroenterologists found the intervention beneficial to their patients, were glad that this resource was available, and were interested in expanding the available services in the future.

The lack of statistically significant change in anxiety and depression scores may reflect several factors. First, compared to the original IMPACT study, which engaged older adults ( $>60$ years old) in CoCM for depression [14], our active subjects in IBD CoCM had relatively low baseline levels of anxiety (mean 8.9, indicating mild anxiety) and depression (mean 10.1 indicating moderate depression). This likely impacted our effect size and contributed to a smaller change in scores from baseline to end of program. 
Second, the sample size was small, largely due to interruptions due to the COVID-19 pandemic. When the pandemic pushed most in-person visits to telemedicine, we no longer had the mechanisms in place to routinely screen patients for mood disorders, in turn relying on patient selfreported symptoms to guide referrals by gastroenterologists. In addition, one-third of patients did not complete the program due to lack of engagement, which was at least partially affected by severe restrictions on in-person visits during the pandemic. On average, these individuals had higher baseline PHQ-9 and GAD-7 scores, suggesting that higher depression and/or anxiety symptom burden may impact continued engagement in the program. This level of disengagement was likely influenced by the care manager's inability to provide in-person meetings and effective patient education resources after the start of the pandemic. In the IMPACT model, the intervention included patient education about depression (video and paper resources) and encouraged the initial visit with the care manager to take place in clinic [14]. The intervention participants also had a mixture of in-person and telephone contacts with the care manager [14]. The model has previously been adapted to an offsite telemedicine-based collaborative care team, which demonstrated improved outcomes compared to practicebased care [23]. In this telemedicine model, access to behavioral interventions (specifically cognitive behavioral therapy) was delivered by videoconferencing and if the patient did not respond to 2 antidepressant trials, a video consultation appointment with the psychiatrist was scheduled. A recent meta-analysis highlighted that telephone compared to face-to-face collaborative care interventions had similar improvements in depressive symptoms; however, antidepressant use decreased with telephone intervention compared to face-to-face delivery [24]. Thus, strictly virtual delivery of CoCM may be effective, but only in situations where the tools to screen patients and deliver care are welldeveloped for virtual use.

Our findings highlight important considerations for future implementation of collaborative care management in this population. The presence of screening for anxiety and depression is a key component of successful collaborative care implementation, as interruption of screening significantly impacted identification of patients with behavioral health concerns and volume of new enrollment. Future suggestions include expanding the capability for electronic screening, which would make screening for anxiety and depression in the IBD clinic more sustainable for telemedicine and in-person visits. In addition, providing patient education resources at time of enrollment highlighting the importance of addressing behavioral health concerns as part of their IBD treatment may help facilitate continued engagement in the program. Particularly given the rise in utilizing telemedicine to deliver care during the pandemic, future studies looking at a telemedicine-based delivered collaborative care intervention in the specialty clinic setting, specifically exploring whether video visits could be an effective replacement for in-person visits, are needed.

In summary, we presented our experience implementing the CoCM pilot in an IBD clinic. While the study is a preliminary pilot with limitations secondary to interruption in-clinic screening and in-person visits due to the COVID-19 pandemic, our findings suggest that CoCM is a feasible care delivery model for this specialty clinic population when mental health screening mechanisms are in place and was well received by the gastroenterologists who treat individuals with IBD.

\section{Acknowledgments}

The authors wish to thank Stephanie Viola, who contributed to the collection of data and all the UNC gastroenterology providers who engaged in the program and provided feedback on the intervention to support future improvements.

\section{Statement of Ethics}

This study protocol was reviewed and approved by UNC Biomedical IRB, reference ID 288720. The study was granted a waiver of informed consent given risk involved in this research is no more than minimal.

\section{Conflict of Interest Statement}

Hans H. Herfarth has served as a consultant for Alivio, AMAG, BMS, ExeGI, Finch, Gilead, Janssen, Lycera, Merck, Otsuka, Pfizer, PureTech, Seres and has received research support from Pfizer and Artizan Biosciences. Millie D. Long has served as a consultant for Pfizer, Janssen, AbbVie, Takeda, Lilly, BMS, Genentech, Roche, Calibr, Salix, Valeant, Prometheus, Theravance, Target PharmaSolutions, and has received research support from Pfizer and Takeda. Christine B. Flicek, Nathaniel A. Sowa, and Spencer D. Dorn have no conflicts of interest to declare.

\section{Funding Sources}

This work was supported by an Innovation Pilot Award program of the UNC Center for Health Innovation. 


\section{Author Contributions}

All authors contributed to the design and conception of the work, provided revisions, and final approval of the manuscript. Christine B. Flicek and Nathaniel A. Sowa additionally contributed to the analysis and interpretation of the data.

\section{Data Availability Statement}

All data generated or analyzed during this study are included in this article. Further inquiries can be directed to the corresponding author.

\section{References}

1 Mikocka-Walus A, Knowles SR, Keefer L, Graff L. Controversies revisited: a systematic review of the comorbidity of depression and anxiety with inflammatory bowel diseases. Inflamm Bowel Dis. 2015;22:752-62.

2 Peppas S, Pansieri C, Piovani D, Danese S, Peyrin-Biroulet L, Tsantes AG, et al. The brain-gut axis: psychological functioning and inflammatory bowel diseases. J Clin Med. 2021;10:377.

3 Goodhand JR, Wahed M, Mawdsley JE, Farmer AD, Aziz Q, Rampton DS. Mood disorders in inflammatory bowel disease: relation to diagnosis, disease activity, perceived stress, and other factors. Inflamm Bowel Dis. 2012;18:2301-9.

4 Graff LA, Walker JR, Bernstein CN. Depression and anxiety in iflammatory bowel disease: a review of comorbidity and management. Inflamm Bowel Dis. 2009;15:1105-18.

5 Jordi SBU, Lang BM, Auschra B, von Känel R, Biedermann L, Greuter T, et al. Depressive symptoms predict clinical recurrence of inflammatory bowel disease. Inflamm Bowel Dis. 2021

6 Kochar B, Barnes EL, Long MD, Cushing KC, Galanko J, Martin CF, et al. Depression is associated with more aggressive inflammatory bowel disease. Am J Gastroenterol. 2018;113: 80-5.

7 Mikocka-Walus A, Pittet V, Rossel JB, von Känel R. Symptoms of depression and anxiety are independently associated with clinical recurrence of inflammatory bowel disease. Clin Gastroenterol Hepatol. 2016;14:829-e1.

8 Limsrivilai J, Stidham RW, Govani SM, Waljee AK, Huang W, Higgins PD. Factors that predict high health care utilization and costs for patients with inflammatory bowel diseases. Clin Gastroenterol Hepatol. 2017; 15:385-e2.
9 Click B, Ramos Rivers C, Koutroubakis IE, Babichenko D, Anderson AM, Hashash JG, et al. Demographic and clinical predictors of high healthcare use in patients with inflammatory bowel disease. Inflam Bowel Dis. 2016;22:1442-9.

10 Regueiro M, Greer JB, Szigethy E. Etiology and treatment of pain and psychosocial issues in patients with inflammatory bowel diseases. Gastroenterology. 2017;152:430-e4.

11 Kristensen MS, Kjærulff TM, Ersbøll AK, Green A, Hallas J, Thygesen LC. Erratum: the influence of antidepressants on the disease course among patients with Crohn's disease and ulcerative colitis-a danish nationwide register-based cohort study. Inflamm Bowel Dis. 2019;25:e152-93.

12 Szigethy EM, Allen JI, Reiss M, Cohen W, Perera LP, Brillstein L, et al. White paper AGA: the impact of mental and psychosocial factors on the care of patients with inflammatory bowel disease. Clinical Gastroenterol Hepatol. 2017;15:986-97.

13 Rubin DT, Ananthakrishnan AN, Siegel CA, Sauer BG, Long MD. ACG clinical guideline: ulcerative colitis in adults. Am J Gastroenterol. 2019;114:384-413.

14 Unützer J, Katon W, Callahan CM, Williams JW, Hunkeler E, Harpole L, et al. Collaborative care management of late-life depression in the primary care setting: a randomized controlled trial. J Am Med Assoc. 2002;288: 2836-45.

15 Archer J, Bower P, Gilbody S, Lovell K, Richards D, Gask L, et al. Collaborative care for depression and anxiety problems. Cochrane Database Sys Rev. 2012.

16 Huffman JC, Niazi SK, Rundell JR, Sharpe M, Katon WJ. Essential articles on collaborative care models for the treatment of psychiatric disorders in medical settings: a publication by the academy of psychosomatic medicine research and evidence-based practice committee. Psychosomatics. 2014;55:109-22.
17 Regueiro MD, McAnallen SE, Greer JB, Perkins SE, Ramalingam S, Szigethy E. The inflammatory bowel disease specialty medical home: a new model of patient-centered care. Inflamm Bowel Dis. 2016;22:1971-80.

18 Spitzer RL, Kroenke K, Williams JB, Löwe B. A brief measure for assessing generalized anxiety disorder: the GAD-7. Arch Intern Med. 2006; 166:1092-7.

19 Kroenke K, Spitzer RL, Williams JB. The PHQ-9: validity of a brief depression severity measure. J Gen Intern Med. 2001;16:606-13.

20 Lewis K, Marrie RA, Bernstein CN, Graff LA, Patten SB, Sareen J, et al. The prevalence and risk factors of undiagnosed depression and anxiety disorders among patients with inflammatory bowel disease. Inflam Bowel Diseases. 2019;25:1674-80.

21 Kroenke K, Spitzer RL, Williams JB, Löwe B. The patient health questionnaire somatic, anxiety, and depressive symptom scales: a systematic review. Gen Hosp Psychiatry. 2010; 32:345-59.

22 Kroenke K. PHQ-9: global uptake of a depression scale. World Psychiatry. 2021;20(1):1356.

23 Fortney JC, Pyne JM, Mouden SB, Mittal D, Hudson TJ, Schroeder GW, et al. Practicebased versus telemedicine-based collaborative care for depression in rural federally qualified health centers: a pragmatic randomized comparative effectiveness trial; 2013. p. 41425.

24 Hudson JL, Bower P, Kontopantelis E, Bee P, Archer J, Clarke R, et al. Impact of telephone delivered case-management on the effectiveness of collaborative care for depression and anti-depressant use: a systematic review and meta-regression. PloS One. 2019;14: e0217948. 